\title{
Enactive Systems \& Computing Mapping the terrain for Human-Computer Interaction research
}

\author{
Alessandro Arpetti, M. Cecília C. Baranauskas \\ Instituto de Computação - Universidade Estadual de Campinas (UNICAMP) \\ Av. Albert Einstein, 1251, Cidade Universitária, Campinas/SP - Brasil, CEP 13083-852 \\ \{alessandro.arpetti,c.baranauskas\} @ic.unicamp.br
}

\begin{abstract}
While the concept of enaction is not new, recent trends on the use of contemporary devices to interact with computational systems have attracted the attention in the field of interaction design. A quick look in the literature reveals different meanings for an enactive system, depending on the knowledge domain. This paper explores the subject by conducting a Systematic Literature Review to construct a big picture on the subject. Results reveal the paths being traced and issues still unexplored that offer opportunities for investigation in the human computer interaction field.
\end{abstract}

\section{Introduction}

Over the past 25 years the concept of "enaction" had a spread in the scientific world, focusing mainly in the field of cognitive science and artificial intelligence (Vernon et al. 2007). In the common language, the term "enaction" derives from the verb "enact" that, in addition to the juridical meaning of establishing by law, means perform a play or put something into practice. Varela, Thompson and Rosch refer to this last meaning of the term to define their concept of cognition, starting the tradition of the enactivism (Varela et al. 1991). For these authors, "cognition is not the representation of a pre-given world by a pre-given mind but is rather the enactment of a world and a mind on the basis of a history of the variety of actions that a being in the world performs" (Ibid. p. 9). This new vision allows the overcoming of the Cartesian dualism of mind and body, reaffirming the importance of the body and the action in relation to both perception and knowledge (Flender 2008).More recently, the concept of enaction was approached to the field of information systems by offering new ways of interaction and involvement of users and systems. The development of new technologies that allow a more physical and immersive modality of interaction brought to the conception of an "enactive system", that "is constituted by dynamically coupled human and technological processes, that is, a dynamic mind-technology embodiment" (Kaipainen et al. 2011). Nevertheless, although the concept of enaction seems to find fertile ground in the Human-Computer Interaction field and in the application of new ways of interaction, a generic search of the string "enactive system" in the main digital libraries returns very few occurrences (Table 1). This result suggests that the literature seems to show a gap between the concept of "enaction", which has a relatively long and well-established history in the field of cognitive science, and its application in the field of information systems. 
Table 1: Results of search for the string "enactive system".

\begin{tabular}{|l|l|}
\hline Source & Results \\
\hline ACM Digital Library & 2 \\
\hline IEEE Xplore & 0 \\
\hline Science Direct & 1 \\
\hline Scopus & 9 \\
\hline Web of Science & 3 \\
\hline & $=15$ (total) \\
\hline & -4 (duplicated) \\
\hline Unique results & $\mathbf{1 1}$ \\
\hline
\end{tabular}

The goal of this paper is to present results of a systematic review about enactive systems in the computing field. This paper is organized as follows: Section 2 describes the research method of the study; Section 3 gives an overview of the results of the study; Section 4 presents a discussion on the reported results. Finally, Section 5 summarizes this work, presents our conclusions and points out future works.

\section{The Method}

This study has been undertaken as a systematic literature review (SLR) following the guidelines for Evidence-based software engineering (EBSE) proposed by Kitchenham (2004). A SLR is a technique used to search for evidence in scientific literature that is conducted in a formal manner, applying well-defined steps, according to a previously elaborated protocol.

This systematic review method is composed by various steps organized in three main phases: phase 1: Planning (specify research questions, develop review protocol, validate review protocol); phase 2: Conducting (identify relevant research, select studies, assess study quality, extract required data, synthesize data), and phase 3 : Reporting (write review report and validate report) (Brereton et al. 2007). During phases 1 and 2 the software "StArt" (Laboratory of Research on Software Engineering (LaPES) - Computing Department of the Federal University of São Carlos (DC/UFSCar) 2010) has been used to provide a support for the compilation and execution of the review. The goal of this review is to summarize the research in the topic of enactive systems and computing, and provide a consistent literature background in order to appropriately position new research activities.

\subsection{Review Questions}

The research questions addressed by this study are: RQ1 - How many studies concerning "enaction" and computing have been published since 2005? RQ2 - What research topics are being addressed? RQ3 - Who is leading these researches? RQ4 - Are these studies theoretical or practical? Do they involve some kind of software application? RQ5 - How the concept of "enaction" is interpreted?

Concerning RQ1, it has been identified the number of publications per year and the journal or conference where the study was published. For RQ2 it has been looked for the main topic area of the study. With respect to RQ3, it has been considered individual researchers, the organization to which researchers were affiliated and the organization country. In relation to RQ4, it has been classified the nature of the study and, eventually, the software applications used or proposed within the research. Finally, for 
RQ5 the definition and use of the "enaction" concept has been observed, both in theoretical and practical applications.

\subsection{Sources and Search Selection Criteria}

The search process started with automatic search in digital libraries. Based on their importance in the computing literature, on their wide range of publication topics and on their popularity, five digital libraries were chosen: ACM Digital Library (http://dl.acm.org), IEEE Xplore (http://ieeexplore.ieee.org), Science Direct (http://www.sciencedirect.com), Scopus (http://www.scopus.com), Web of Science (https://www.webofknowledge.com). Since the search for the string "enactive system" returns, as mentioned above, a few number of results, a big concern for the definition of the search string was to add a greater number of results and to provide a broader basis for answering the research questions. The result was to select all the publications subsequent to 2004 that contained in the title, in the abstract or in the keywords at least one of the terms: "enaction", "enactive" or "enactivism".

\subsection{Sources and Search Selection Criteria}

For the definition of inclusion and exclusion criteria it has been taken into account the requirements due to the research questions and the linguistic possibilities of the researchers who carried out the selection. To be included in the review, the articles must be: peer-reviewed; published between Jan 1, 2005 and September 30, 2015; written in English, French, Italian, Portuguese or Spanish; related to the computing area; computing and Enaction must not have a marginal role in the study. Excluded articles are those that, in addition to those that did not meet any of the criteria mentioned above, were: short articles; duplicated studies.

\subsection{Data Extraction}

After the selection phase, it was the turn of to the data extraction process that was performed by reading the abstract and screening the full-text of each one of the selected studies. The data extracted from each study were: 1) Paper Information (Title, Year, Authors, Affiliation, Country, Source type [Journal, Conference]); 2) Research Area; 3) Topic; 4) Research type (Theoretical, Empirical); 5) Use or propose any tool? (Yes, No); 6) If use a technological tool: what type of tool? (Web application, Simulation software, Data visualization, NUI, Ontological tool, Robotics); 7) Interpretation of the "Enaction" concept (as body presence, as system interaction, as interface modality, as common vocabulary, as Varela's definition).

\section{Results}

This section presents the results of the review, based on studies selection and data extraction processes. Firstly, the automatic search was conducted at each digital library. Then, an iterative process was applied to exclude duplicate and not relevant papers.

The result of the automatic search reached 1268 studies, mainly found in Scopus $(52.6 \%)$ and Web of Science (38.3\%) (Figure 1). With the selection process, 594 duplicate results were detected and 602 studies were rejected, based on inclusion/exclusion criteria and full-text screening. As final result of the selection process, 72 studies were selected and admitted to the data extraction process. 
Concerning the type of publication, 27 studies were published in conference proceedings $(37 \%)$, while 45 papers in journals (63\%). As regards of the temporal distribution of the studies, it is possible to notice a growing linear trend, characterized by an irregular distribution with a greater or lesser number of publications depending on the year (Figure 2).

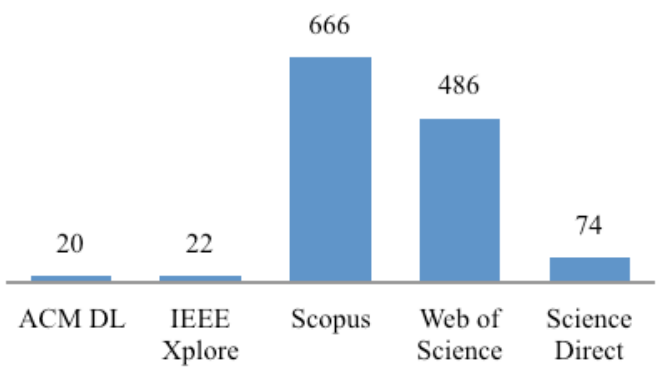

Figure 1: Automatic search results per source.

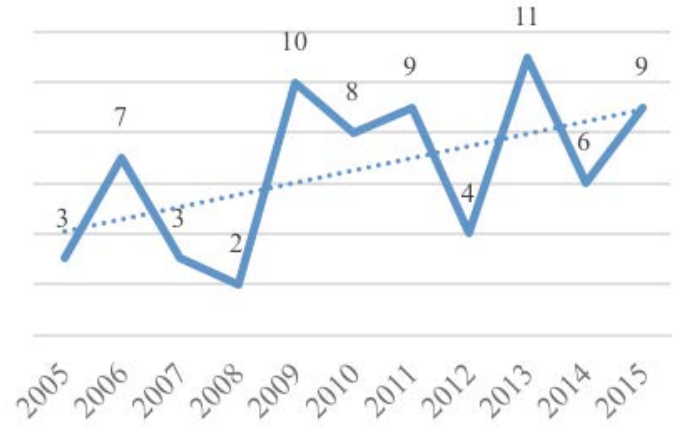

Figure 2: Distribution of publications per year.

The papers presented in conferences are distributed among 21 conferences (Table 2). Only 4 conferences show the presence of more than one paper, with the "Artificial Intelligence and Simulation of Behavior" (AISB) and the "ACM SIGCHI Creativity and Cognition" (SIGCHI CC) conferences with 3 papers, the "American Society Of Mechanical Engineers" (ASME) and the "International Society for Optics and Photonics" (IS\&T/SPIE) conferences with 2 papers.

Table 2: List of Conferences.

\begin{tabular}{|l|l|l|l|l|l|}
\hline AISB & 3 & CASYS & 1 & EDU LEARN & 1 \\
\hline SIGCHI CC & 3 & CogInfoCom & 1 & ICDL & 1 \\
\hline ASME & 2 & CORIA & 1 & ICHIM & 1 \\
\hline IS\&T/SPIE & 2 & CSCL & 1 & IROS & 1 \\
\hline ALIFE & 1 & CW & 1 & T4E & 1 \\
\hline BICA & 1 & CYBCONF & 1 & WCECS & 1 \\
\hline CAiSE-DC & 1 & DEST & 1 & WCETR & 1 \\
\hline
\end{tabular}

These conferences are related to 10 research fields (Table 3 ) and it is notable that $48.1 \%$ of the conferences concern AI, Robotics and Cybernetics, while 40.7\% are divided between Education, Art and Information Systems.

Table 3: Conferences research fields.

\begin{tabular}{|l|l|l|l|}
\hline Artificial Intelligence & 7 & Cybernetics & 2 \\
\hline Education & 4 & Cognitive science & 1 \\
\hline Robotics & 4 & Computing & 1 \\
\hline Art - Technology & 3 & Optical sciences & 1 \\
\hline Information System & 3 & Virtual reality & 1 \\
\hline
\end{tabular}


The number of journals in which the papers are published is 33, with 8 journals presenting more than one publication. Among them there are "Kybernetes" with 4 papers, "Lecture Notes in Artificial Intelligence" and "Leonardo" with 3 papers, the "Journal of E-Learning and Knowledge Society", "Virtual Reality", "AI \& Society", "Constructivist Foundations" and "Digital Creativity" with 2 papers (Table 4).

Table 4: List of Journals.

\begin{tabular}{|l|l|}
\hline Kybernetes & 4 \\
\hline Lecture Notes in Artificial Intelligence & 3 \\
\hline Leonardo & 3 \\
\hline Journal of E-Learning and Knowledge Society & 2 \\
\hline Virtual Reality & 2 \\
\hline AI \& Society & 2 \\
\hline Constructivist Foundations & 2 \\
\hline Digital Creativity & 2 \\
\hline Adaptive Behavior & 1 \\
\hline Artificial Life \& Technology & 1 \\
\hline Automated Systems Based on Human Skill and Knowledge & 1 \\
\hline Cognitive Computation & 1 \\
\hline Computer-Aided Design and Applications & 1 \\
\hline Contemporary Music Review & 1 \\
\hline Educação em Revista & 1 \\
\hline Educational Research & 1 \\
\hline Educational Technology Research and Development & 1 \\
\hline Emotion Modeling & 1 \\
\hline Frontiers in Artificial Intelligence and Applications & 1 \\
\hline IEEE Transactions on Haptics & 1 \\
\hline IEEE Transactions on Evolutionary Computation & 1 \\
\hline International Journal of Interactive Design and Manufacturing & 1 \\
\hline International Journal of Machine Consciousness & 1 \\
\hline Journal of Experimental \& Theoretical Artificial Intelligence & 1 \\
\hline Journal of Interactive Learning Research & 1 \\
\hline Journal of New Music Research & 1 \\
\hline Journal of Research on Technology in Education (International Society for Technology in & 1 \\
\hline Education) & 1 \\
\hline L'Année psychologique & 1 \\
\hline Minds and Machines & 1 \\
\hline Personal Ubiquitous Computing & 1 \\
\hline Philosophy \& Technology & 1 \\
\hline PsychNology Journal & 1 \\
\hline Systems Research and Behavioral Science & \\
\hline
\end{tabular}

The list of research fields related to these journals amounts to 11 and, as for the conferences, almost half of the papers are published in the AI, Robotics and Cybernetics 
field (42.2\%), while the absence of Journals related to the Information Systems field is replaced by a bigger number of publications in Psychology and Cognitive Science fields $(15.6 \%)$ (Table 5).

Table 5: Journals research fields.

\begin{tabular}{|l|l|l|l|}
\hline Artificial Intelligence & 14 & Design & 2 \\
\hline Education & 7 & Music & 2 \\
\hline Art - Technology & 5 & Virtual reality & 2 \\
\hline Cybernetics & 4 & Computing & 1 \\
\hline Psychology & 4 & Robotics & 1 \\
\hline Cognitive science & 3 & & \\
\hline
\end{tabular}

The review holds contributions from 22 countries located in all the continents (Figure 3 ), but with a concentration in the Americas (23\%) and Europe (68\%) (Figure 4).It is interesting to note that only five countries (UK, Italy, France, Sweden and USA) gathered a total of 53 publications, accounting for $73.6 \%$ of the total.
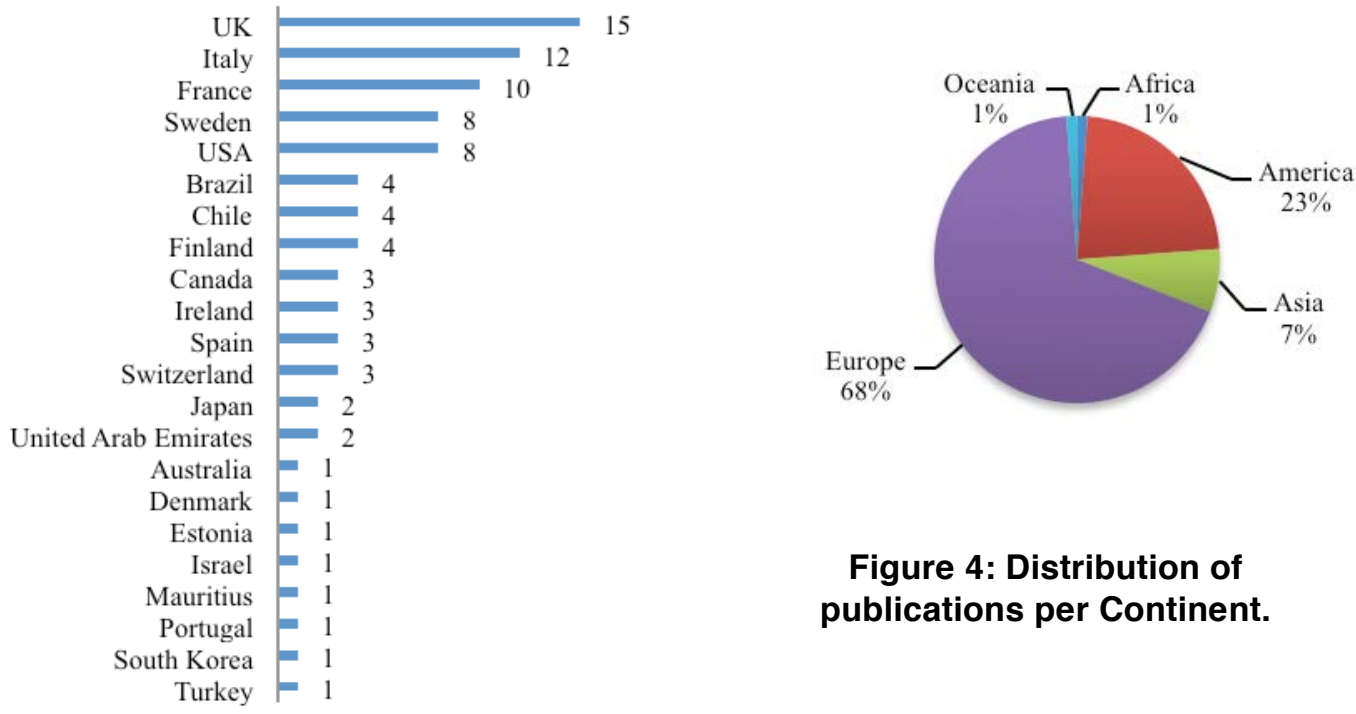

Figure 3: Distribution of publications per Country.

The list of authors appears quite varied, with a total of 176 and an average of 2.4 authors per paper. 28 authors are present in more than one study, with 21 authors with 2 papers, 5 with 3 papers and 2 authors with 4 papers (Figure 5). Even for organizations to which the authors are affiliated, it is possible to notice a wide variety, with 82 different entities. Between them, 17 are repeated more than once. The most active organizations are the Informatics Research Centre of the University of Skovde, in Sweden, the Polytechnic of Milano, in Italy, the Department of Informatics of the University of Sussex, in UK and the Industrial Engineering Department of the University of Santiago de Chile, in Chile (Figure 6). 


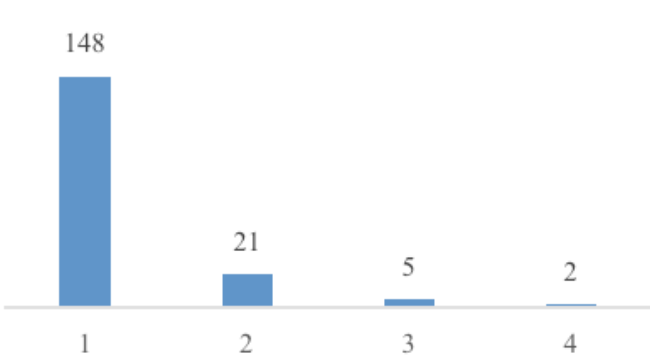

Figure 5: Number of papers per author.

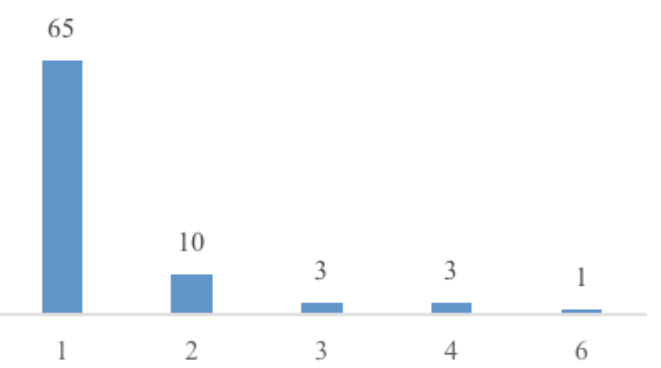

Figure 6: Number of papers per organization.

In relation to the research areas, 17 fields have been identified (Figure 7), with a primary focus of studies in Artificial Intelligence (AI) and Robotics (27.8\%), Educational Technology (20.8\%) and Human-Computer Interaction (HCI - 18.1\%).

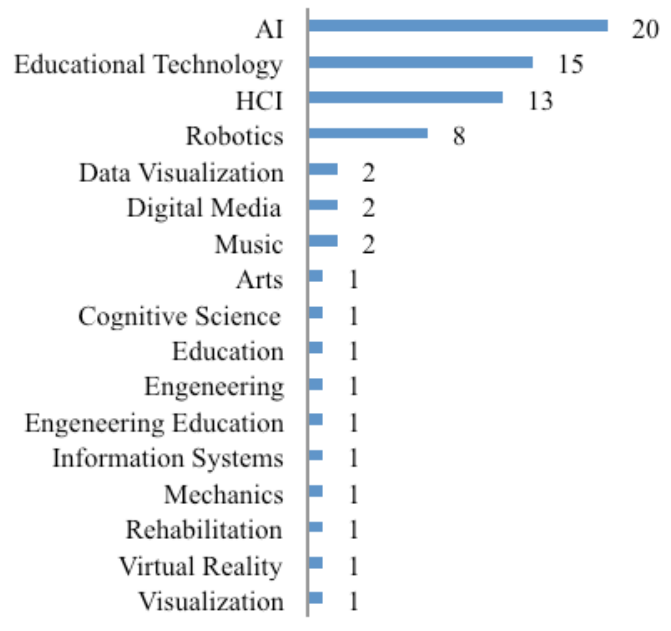

Figure 7: Research areas of publications.

The topics of the papers are more dispersed and it was possible to identify 53 different subjects, with a slight concentration for the modeling of emotions, for virtual reality, for autonomous and conscious cognitive systems, and for different types of interfaces (Table 6).

Table 6: Topics of selected studies.

\begin{tabular}{|l|l|l|l|}
\hline Topic & $\begin{array}{l}\text { N. of } \\
\text { studies }\end{array}$ & Topic & $\begin{array}{l}\text { N. of } \\
\text { studies }\end{array}$ \\
\hline $\begin{array}{l}\text { Computational modeling of } \\
\text { emotion }\end{array}$ & 6 & Digital narratives & 1 \\
\hline Virtual reality & 4 & Educational methods & 1 \\
\hline Enactive interfaces & 3 & Enactive systems & 1 \\
\hline Machine consciousness & 3 & Engineering learning & 1 \\
\hline Multimodal interfaces & 3 & Game-based learning & 1 \\
\hline Autonomous cognitive systems & 2 & Gesture analysis & 1 \\
\hline
\end{tabular}




\begin{tabular}{|l|l|l|l|}
\hline Decisional information system & 2 & Haptic interfaces & 1 \\
\hline Motion capture & 2 & Human-robot interaction & 1 \\
\hline Second-order cybernetics & 2 & Instructional design & 1 \\
\hline Affordances & 1 & Interaction & 1 \\
\hline Appropriation and perception & 1 & Interactive machine learning & 1 \\
\hline Augmented instruments & 1 & Learning organizations & 1 \\
\hline Autonomous learning & 1 & Mathematics education & 1 \\
\hline Autonomous robotics & 1 & Motor skills & 1 \\
\hline Bioart & 1 & Multimodal resources & 1 \\
\hline Bio-machine hybrids & 1 & Narrative media & 1 \\
\hline Blended learning & 1 & Ontogeny & 1 \\
\hline Body schema & 1 & Phenomenology & 1 \\
\hline Conceptual modeling & 1 & Philosophy of AI & 1 \\
\hline Cybernetics & 1 & Physical education & 1 \\
\hline Design Tools & 1 & Situated cognition & 1 \\
\hline Digital classroom & 1 & Social robots & 1 \\
\hline Digital controllers & 1 & $\begin{array}{l}\text { System design and } \\
\text { evaluation }\end{array}$ & 1 \\
\hline Digital environment design & 1 & Tool design and evaluation & 1 \\
\hline Digital game creation & 1 & Tool development & 1 \\
\hline Digital game design & 1 & Visual Music & 1 \\
\hline Digital game learning & 1 & & \\
\hline
\end{tabular}

Analyzing the list of keywords found in the selected papers (Figure 8), it is possible to notice that the more frequent terms are exactly the principal terms of this review, but with no relationship between them, except for the paper by Domingues et al. (2014) that presents the keyword "enactive systems". Among the other keywords, is noteworthy the wide presence of the term "learning" and the less obvious presence of the terms "creativity" and "design". Finally, from the technological point of view, it is relevant the presence of the terms "haptic" and "virtual" "reality".

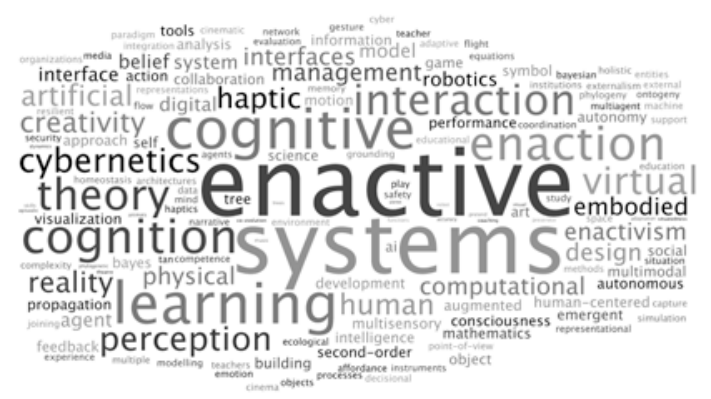

Figure 8: Keywords tag cloud.

The type of research for the selected studies is balanced, with 34 theoretical (47\%) and 38 empirical studies (53\%). The same proportion is maintained for studies that use or present a technological tool $(47 \%)$ and those that do not $(53 \%)$, but with a list of different publications in each group, since 4 practical studies do not use tools and vice versa. This condition is explained by the fact that some empirical studies do not use technological tools, but traditional elements during the experiments (printed materials, physical objects, etc.), while some theoretical studies use some technologies to explain or illustrate phenomena, especially in robotics and cybernetics. As for the studies that use or present a technological tool, most of them are based on the use of NUI (42.3\%), 
often coupled with virtual simulation technologies $(25 \%)$. The application in robotics is fairly common (17.3\%), while less frequent is the presence of Data visualization $(7.7 \%)$ or Ontological tools (7.7\%) (Figure 9). Finally, in relation to the use and the interpretation of the "enaction" concept, it is possible to notice that the majority of the selected studies $(61.9 \%)$ follow the original definition of Varela et al. The remaining studies $(38.1 \%$ ) adopt an interpretation more linked to an embodied modality of interaction in which the presence of the body is related to new typology of interfaces and information systems (Figure 10).

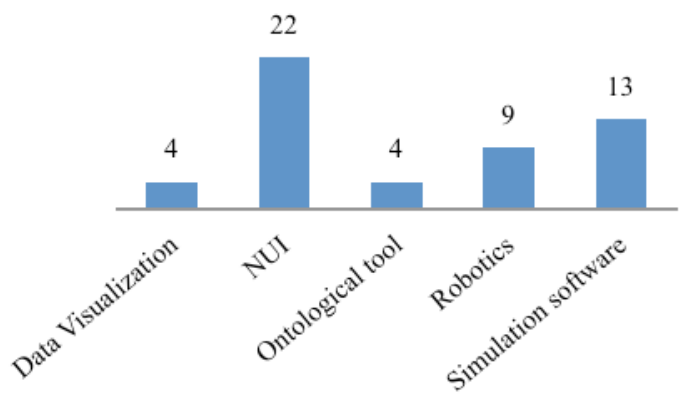

Figure 9: Kind of technological tools.

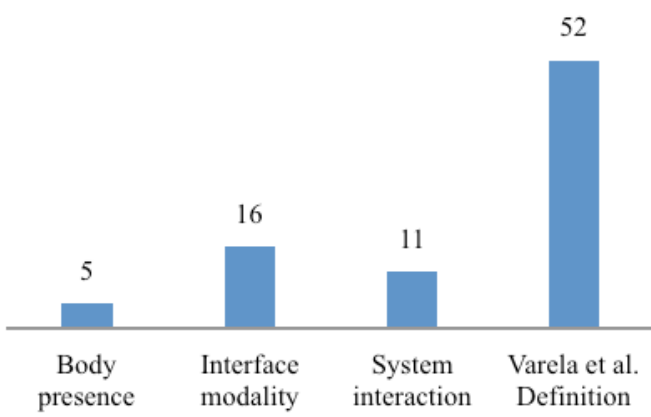

Figure 10: Interpretation of the "enaction" concept.

For example, an interpretation of the concept of enaction as body presence is that present in Di Tore et al. (2013) for whom the knowledge is codified in the form of motor responses and acquired in the action. An interpretation as an interface modality is offered instead by Froese et al. (2012), whom interprets the interfaces as augmented sense-making that serve as a transparent medium for augmenting our natural skills of interaction with the world. An interpretation of the enaction as system interaction is offered by Fedeli \& Rossi (2011) for whom a system transforms itself thanks to the interaction between different subjects and to the mind-body-artifact-world continuum. Finally, an example of interpretation as the original Varela definition is proposed by Pugliese \& Lehtonen (2011) that synthesize the concept as a continuous two-directional loop of bodily interaction. This process is a feedback loop: the actions performed by the enactor affect the medium that in turn affects the following actions of the enactor. Focusing the attention on the 13 papers related to the Human-Computer Interaction area, we can notice a bigger concentration in the latest 5 years, with a balanced distribution between journals and conferences (Table 7).

Table 7: Studies related to $\mathrm{HCl}$.

\begin{tabular}{|l|l|l|}
\hline Paper & Journal & Conference \\
\hline (Bordegoni \& Cugini 2013) & $\mathrm{x}$ & \\
\hline (Bordegoni \& Cugini 2009) & & $\mathrm{x}$ \\
\hline (Davis, Comerford, et al. 2015) & & $\mathrm{x}$ \\
\hline (Davis, Hsiao, et al. 2015) & & $\mathrm{x}$ \\
\hline (Flint \& Turner 2015) & $\mathrm{x}$ & \\
\hline (Froese et al. 2012) & $\mathrm{x}$ & \\
\hline (Kaipainen et al. 2011) & $\mathrm{x}$ & \\
\hline (Luyat \& Regia-Corte 2009) & $\mathrm{x}$ & \\
\hline (Pacheco \& Souza-Concilio 2013) & & $\mathrm{x}$ \\
\hline
\end{tabular}




\begin{tabular}{|l|l|l|}
\hline (Pugliese \& Lehtonen 2011) & x & \\
\hline (Saidali et al. 2009) & & x \\
\hline (Todaro et al. 2007) & & x \\
\hline (van der Linden et al. 2011) & & x \\
\hline
\end{tabular}

Topics of these papers are related to three main subjects: Interfaces (enactive interfaces, haptic interfaces, multimodal interfaces); Design (system design and evaluation, tool design and evaluation, digital environment design, enactive systems); Perception (appropriation and perception, motion capture, affordances).

Within these studies, the concept of enaction is interpreted as system interaction (4 studies), interface modality (6 studies) and as the definition of Varela et al. (8 studies). 4 of these studies are theoretical, while the other 9 are mainly practical and empirical. All the practical studies use or propose a technological tool; among them 3 simulation software, 1 data visualization and 7 NUI. Among the theoretical studies, one of them uses a software game.

\section{Discussion}

Although the initial search for "enactive system" returned few results, a more thorough search for "enaction", "enactive" or "enactivism", returned an expressive number of papers. It is worth noticing that most papers come from journals, where more mature research is usually presented. In the last ten years, a growing presence of the subject was detected. Most contributions come from Europe, mainly associated to the fields of Artificial Intelligence, Educational Technology and HCI. The Significant presence in AI seems related to the development of a new paradigm in robotics. The main topics addressed the subject associating it to computational modeling of emotion and virtual reality. There is a good balance among the papers between theoretical and empirical contributions, and also among the contributions that propose or use tools and those that do not. For those studies that embed tools, those usually refer to NUI (natural user interfaces). Experimentations with NUI and virtual reality are located especially in the educational technology field.

Within the field of Human Computer Interaction, the studies address issues mainly related to the design and evaluation of new types of interfaces (enactive, multimodal, haptics), and to an in-depth analysis of perception in relation to enaction and design solutions. This set of facts gives us a snapshot of the use of enactive systems in the computing field research in the last ten years.

\section{Conclusions}

The enaction may be understood as the quality of interactive systems that allow organization of knowledge obtained through a circular action. Examples are interfaces that couple a human with a machine to do things usually done unaided, such as shaping a three-dimensional object using multiple modality interactions with a data base or using interactive video to allow a student to visually engage with mathematics concepts (Bordegoni 2010; Tall et al 2008). Although several understandings for the term exist, depending on the knowledge domain area, the SLR revealed a wider adherence to the traditional concept of "enaction", as proposed by Varela et al (1991). Within these coordinates, the concept of enaction links biology and phenomenology, emphasizing the interaction of the embodied organism with its environment. It is not a question of the 
separation of the representation and the action in an objective world, but it is the same activity of the embodied organism that produces a world of meaning (Nehaniv et al. 2013). The presence of the majority of studies in Europe and America seems to suggest the complexity and multidisciplinary nature of the topic that, based on a large philosophical background, is positioned in a breaking line with the tradition. These factors, as well as the cultural conditions and freedom in experimenting with new solutions, can strongly influence the understanding and design of enactive systems. Moreover, the SLR exposed a lack of studies regarding the investigation of the "enaction" concept in relation to the web or social systems. This indicates an opportunity for research, especially in the field of HCI that may lead to innovative ways of understanding the social phenomena mediated by technology. Further work involves deepening the subject to include specificities of different knowledge domains to the concept and use of enactive systems.

\section{Acknowledgements}

This work was conducted during a PNPD/CAPES scholarship at the University of Campinas.

\section{References}

Bordegoni, M., 2010. Design tools based upon enactive interfaces. In Shuichi Fukuda, ed. Emotional Engineering: Service Development. Springer. pp. 78.

Bordegoni, M. \& Cugini, U., 2009. Multimodal Perception-Action Interaction for the Exploitation of Enactive Knowledge. In Volume 2: 29th Computers and Information in Engineering Conference, Parts A and B. ASME, pp. 983-990.

Bordegoni, M. \& Cugini, U., 2013. Tools for Industrial Design: From Barriers to Enablers of Creativity. Computer-Aided Design and Applications.

Brereton, P. et al., 2007. Lessons from applying the systematic literature review process within the software engineering domain. Journal of Systems and Software, 80(4), pp.571-583.

Davis, N., Comerford, M., et al., 2015. An Enactive Characterization of Pretend Play. Proceedings of the 2015 ACM SIGCHI Conference on Creativity and Cognition $C \& C^{\prime} 15$, pp.275-284.

Davis, N., Hsiao, C., et al., 2015. Drawing Apprentice: An Enactive Co-Creative Agent for Artistic Collaboration. In Proceedings of the 2015 ACM SIGCHI Conference on Creativity and Cognition. pp. 185-186.

Domingues, D. et al., 2014. Embodiments, visualizations, and immersion with enactive affective systems. In Proc. SPIE 9012, The Engineering Reality of Virtual Reality 2014.

Fedeli, L. \& Rossi, P.G., 2011. A study of teacher/student relations in a formal face-toface niversity context supported by online tools within an enactive pproach. Procedia - Social and Behavioral Sciences, 28, pp.673-678.

Flender, C., 2008. Ecological Modelling of Information Systems. In Proceedings of CAiSE-DC. pp. 40-52. 
Flint, T.\& Turner, P., 2015. Enactive appropriation. Ai \& Society, pp.1-9.

Froese, T. et al., 2012. The enactive torch: A new tool for the science of perception. IEEE Transactions on Haptics, 5(4), pp.365-375.

Kaipainen, M. et al., 2011. Enactive Systems and Enactive Media: Embodied HumanMachine Coupling beyond Interfaces. Leonardo, 44(5), pp.433-438.

Kitchenham, B., 2004. Procedures for performing systematic reviews. Keele, UK, Keele University, 33(TR/SE-0401), p.28.

Laboratory of Research on Software Engineering (LaPES) -Computing Department of the Federal University of São Carlos (DC/UFSCar), 2010. StArt (State of Art through Systematic Review). Available at: http://lapes.dc.ufscar.br/tools/start_tool.

van der Linden, J. et al., 2011. Haptic reassurance in the pitch black for an immersive theatre experience. In Intelligent Robots and Systems (IROS), 2010 IEEE/RSJ International Conference on. pp. 4686-4691.

Luyat, M. \& Regia-Corte, T., 2009. The affordances : From James Jerome Gibson to the recent formal models of the concept. Annee Psychologique, 109(2), pp.297-332.

Nehaniv, C.L. et al., 2013. Interaction and experience in enactive intelligence and humanoid robotics. Artificial Life (ALIFE), 2013 IEEE Symposium on, pp.148-155.

Pacheco, B. a. \& Souza-Concilio, I. a., 2013. Multimodal interfaces: An enactive approach. In 4th IEEE International Conference on Cognitive Infocommunications, CogInfoCom 2013 - Proceedings. pp. 51-58.

Pugliese, R. \& Lehtonen, K., 2011. A framework for motion based bodily enaction with virtual characters. Lecture Notes in Computer Science (including subseries Lecture Notes in Artificial Intelligence and Lecture Notes in Bioinformatics), 6895 LNAI, pp.162-168.

Saidali, Y. et al., 2009. Aid to the interpretation of legal documents: A user-centered approach. In CORIA 2009 - Conférence en Recherche d'Information et Applications. pp. 457-468.

Todaro, E. et al., 2007. The Hand Dance: A Didactic Performance Platform. In International Cultural Heritage Informatics Meeting (ICHIM07): Proceedings,

Di Tore, P.A., Discepolo, T. \& Di Tore, S., 2013. Natural user interfaces as a powerful tool for courseware design in physical education. Journal of E-Learning and Knowledge Society, 9(2), pp.109-118.

Tall, D, Smith D., Piez C. 2008. Enactive control. In Mary Kathleen Heid, Glendon W. Blume, eds. Research on Technology and the Teaching and Learning of Mathematics. Information Age Publishing Inc. pp. 213.

Varela, F.J., Thompson, E. \& Rosch, E., 1991. The Embodied Mind: Cognitive Science and Human Experience. An International Journal of Complexity and, 1992, p.328.

Vernon, D. et al., 2007. A Survey of Artificial Cognitive Systems : Implications for the Autonomous Development of Mental Capabilities in Computational Agents. , 11(2), pp.151-180. 